\title{
Mass estimate of the XTE J1650-500 black hole from the extended orbital resonance model for high-frequency QPOs (Research Note)
}

\author{
P. Slaný and Z. Stuchlík
}

\author{
Institute of Physics, Faculty of Philosophy and Science, Silesian University in Opava, Bezručovo nám. 13, 74601 Opava, \\ Czech Republic \\ e-mail: petr.slany@fpf.slu.cz
}

Received 6 June 2008 / Accepted 20 September 2008

\begin{abstract}
Context. XTE J1650-500 is a Galactic black-hole binary system for which at least one high-frequency QPO at $250 \mathrm{~Hz}$ has been reported. Moreover there are indications that the system harbours a near-extreme Kerr black hole with a spin $a_{*} \simeq 0.998$ and mass $M_{\mathrm{BH}} \lesssim 7.3 M_{\odot}$. Recently it was discovered that the orbital 3-velocity of test-particle (geodesical) discs orbiting Kerr black holes with a spin $a_{*}>0.9953$, analyzed in the locally non-rotating frames, reveals a hump near the marginally stable orbit. It was suggested that the hump could excite the epicyclic motion of particles near the ISCO with frequencies typical for high-frequency QPOs. The characteristic frequency of the hump-induced oscillations was defined as the maximal positive rate of change of the LNRF-related orbital velocity with the proper radial distance. If the characteristic "humpy frequency" and the radial epicyclic frequency are commensurable, strong resonant phenomena are expected.

Aims. We apply the idea of hump-induced oscillations in accretion discs around near-extreme Kerr black holes to estimate the blackhole mass in the XTE J1650-500 binary system.

Methods. For the Kerr black hole with spin $a_{*} \simeq 0.9982$ the characteristic "humpy frequency" and the radial epicyclic frequency are in the ratio 1:3 at the orbit where the positive rate of change of the LNRF-related orbital velocity with the proper radial distance is maximal. Identifying the radial epicyclic frequency with the observed $250 \mathrm{~Hz}$ QPO, we arrive at the mass of the black hole. In this method the ratio of frequencies determines the spin (and vice versa), and the values of the frequencies determine the black-hole mass. Results. The mass of the Kerr black hole in XTE J1650-500 binary system is estimated to be around 5.1 $M_{\odot}$.
\end{abstract}

Key words. accretion, accretion disks - black hole physics - relativity - X-rays: individuals: XTE J1650-500

\section{Introduction}

The Galactic X-ray transient binary black-hole system XTE $\mathrm{J} 1650-500$ is a source in which a near-extreme stellar-mass Kerr black hole is assumed (another one is, e.g., the microquasar GRS 1915+105). Miller et al. (2002) observed the source with the XMM-Newton/EPIC-pn device close to the peak of its outburst in September 2001, and found a broad, skewed Fe K $\alpha$ emission line in its spectrum, indicating a stellar-mass Kerr $\mathrm{BH}$ with near-maximal angular momentum (spin) close to the value $a_{*} \simeq 0.998$. In fact, the spin $a_{*}=0.998$ corresponds to the well-known "Thorne limit", which is the upper limit on spin of the "Laor" model (Laor 1991) used by Miller et al. to fit the line. The "Thorne limit" is an important consequence of theoretical considerations of how photons coming from the inner part of a geometrically thin, geodesical accretion disc around the Kerr black hole influence a spin-up of the hole induced by matter accreting into the hole. Depending on the photon emission law the limiting value slightly differs from the "canonical value" $a_{*} \simeq 0.998$, being 0.9978 for isotropic emission and 0.9982 for the electron-scattering emission law, see Thorne (1974) for more details. Evidence of such a high spin in XTE J1650-500 also was found in three observations with BeppoSAX during the same outburst, which were performed just before and after the XMMNewton observation, see Miniutti et al. (2004). On the other hand Done \& Gierliński (2006) suggests that if the absorption features in the optically thick hot corona are taken into account, the inferred relativistic smearing of the $\mathrm{K} \alpha$ line can be substantially reduced to be compatible with the conception of a disc truncated at $\sim 10 r_{\mathrm{g}}\left(r_{\mathrm{g}}=G M_{\mathrm{BH}} / c^{2}\right.$ is the gravitational radius). This is accomplished by the evaporating hot inner accretion flow, and thus gives no information on the black-hole spin. In this paper we analyze one consequence of the possibility that the black hole in XTE J1650-500 is very rapidly rotating.

Homan et al. (2003) report the detection of a high-frequency variability in X-ray flux from XTE J1650-500, analyzing data from the Rossi X-Ray Timing Explorer obtained from September to November 2001. During transition of the source from the hard to the soft state, the high-frequency QPO around $250 \mathrm{~Hz}$ (with a maximum observed frequency of $270 \mathrm{~Hz}$ ) was detected. Homan et al. (2003) also report broad high-frequency features around $50 \mathrm{~Hz}, 109 \mathrm{~Hz}$, and $168 \mathrm{~Hz}$, among which the feature around $50 \mathrm{~Hz}$ is remarkably stable. Similarly, Kalemci et al. (2003) also report broad features near $80 \mathrm{~Hz}$ (but with a quite large error of $\sim 50 \%$ ) and $25 \mathrm{~Hz}$, but no high-frequency QPO. It is not clear whether this $80 \mathrm{~Hz}$ peak is the same as the $50 \mathrm{~Hz}$ peak found by Homan et al. (2003). Currently, we can only say that the data reveal some broad high-frequency features with frequencies of tens of $\mathrm{Hz}$, and possibly one high-frequency QPO around $250 \mathrm{~Hz}$.

Subsequently, Orosz et al. (2004) published their analysis of the $R$-band photometry of the binary system XTE J1650-500, 


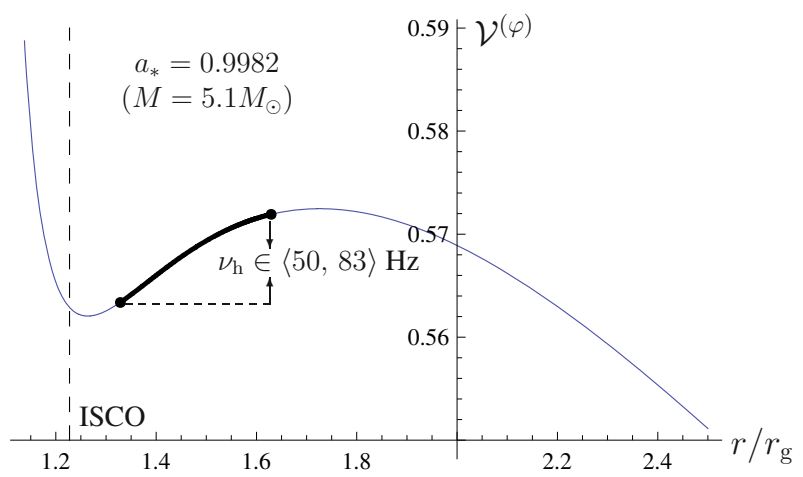

Fig. 1. Orbital velocity profile along equatorial circular geodesics (in units of $c$ ) determined by the locally non-rotating frames in the case of a very rapidly rotating Kerr black hole with a dimensionless spin $a_{*}=0.9982$. The positive part of the radial gradient of $\mathcal{V}^{(\varphi)}$ is located close to but above the innermost stable circular orbit (ISCO). The thick part corresponds to the region where the humpy frequency, determined for the $5.1 M_{\odot}$ black hole, reaches the values from the interval $50-83 \mathrm{~Hz}$, corresponding to observed broad high-frequency features in XTE J1650-500.

determining the optical mass function, $f(M)=(2.73 \pm 0.56) M_{\odot}$, and a lower limit to the inclination of the system of $50^{\circ}$. The results give an upper limit to the mass of the black hole in XTE J1650-500 of $M_{\mathrm{BH}} \lesssim 7.3 M_{\odot}$ (Orosz et al. 2004).

We summarize that the spectral and timing properties of the X-ray transient black-hole candidate XTE J1650-500 suggest that this source hosts a stellar-mass (and possibly near-extreme) Kerr BH in its center.

\section{Aschenbach effect in thin, geodesical discs}

In the case of very rapidly rotating Kerr black holes (with the dimensionless $\operatorname{spin} a_{*}=c J / G M_{\mathrm{BH}}^{2}>0.9953 ; J$ is the blackhole angular momentum), a test particle orbital 3-velocity $\mathcal{V}^{(\varphi)}$, defined by appropriate projections of the particle's 4-velocity $U^{\mu}$ onto the LNRF-tetrad (Bardeen et al. 1972) ${ }^{1}$, reveals a nonmonotonic profile in the equatorial plane (Aschenbach 2004). Stuchlík et al. (2005) shown that the analogous "humpy" behaviour of the $\mathcal{V}^{(\varphi)}$ appears also in the non-geodesic motion of a test perfect fluid orbiting near-extreme Kerr black holes with spin $a_{*}>0.9998$ in marginally stable thick discs (tori), characterized by a uniform distribution of the specific angular momentum, $\ell(r, \theta)=-U_{\varphi} / U_{t}=$ const $^{2}$. In both geometrically thin and thick accretion discs, the positive part of $\partial \mathcal{V}^{(\varphi)} / \partial r$ is confined to the ergosphere around the black hole, however it is located above the innermost stable circular orbit (ISCO) in the equatorial plane $(\theta=\pi / 2)$; see Fig. 1 . Therefore local processes in the inner part of accretion discs around near-extreme Kerr black holes could carry a signature of the orbital velocity hump.

Aschenbach (2004) introduced a characteristic (critical) frequency of any process connected to the velocity hump by the maximum positive slope of the orbital velocity $\mathcal{V}^{(\varphi)}$ given as a function of the B-L radius $r$. Its coordinate-independend

\footnotetext{
${ }^{1}$ In rotating spacetimes a rotation of the space, causing so-called "dragging of inertial frames", is superposed on the orbital motion of matter in the disc, as viewed from infinity. Locally non-rotating frames (LNRF) are dragged along with the spacetime, thus the LNRF should reveal local orbital properties of the disc in the clearest way, as the static observers do in non-rotating spacetimes.

${ }^{2}$ Standard Boyer-Lindquist (B-L) coordinates $(t, r, \theta, \varphi)$ are used.
}

definition, using the maximal positive rate of change of $\mathcal{V}^{(\varphi)}$ with the proper radial distance $\tilde{r}$,

$v_{\text {crit }}^{\tilde{r}}=\left.\frac{\partial \mathcal{V}^{(\varphi)}}{\partial \tilde{r}}\right|_{\max }, \quad \mathrm{d} \tilde{r}=\sqrt{g_{r r}} \mathrm{~d} r$

was suggested by Stuchlík et al. (2004). Relating this locally defined characteristic frequency $v_{\text {crit }}^{\tilde{r}}$ to a static observer at infinity, we obtain so-called "humpy frequency" (Stuchlík et al. 2007a)

$v_{\mathrm{h}}=\sqrt{-\left(g_{t t}+2 \omega g_{t \varphi}+\omega^{2} g_{\varphi \varphi}\right)} v_{\text {crit }}^{\tilde{r}}$,

where $g_{\mu \nu}$ are metric coefficients of the Kerr geometry (in B-L coordinates), and $\omega=-g_{t \varphi} / g_{\varphi \varphi}$ is the angular velocity of the LNRF (Bardeen et al. 1972). However, the frequency characterizing the Aschenbach effect in the disc need not be fixed and defined strictly as the maximal gradient of the orbital velocity in the proper radial distance, Eq. (1). Rather we expect that the characteristic frequency can slightly change and be shifted from this maximal value. On the other hand, at the orbit where it is maximal, i.e. at the so-called "humpy radius", the Aschenbach effect is the strongest. Due to possible changes of the humpy frequency the Aschenbach effect in the disc should not be characterized by a narrow frequency peak but rather by one relatively wide and not very strong QPO with a centroid frequency of several tens $\mathrm{Hz}$ (for stellar mass Kerr black holes).

Aschenbach (2004) also gave a heuristic assumption on the possible excitation of a particle's epicyclic motion in the inner part of geodesic discs by the orbital velocity hump. The epicyclic motion is characterized by the radial and vertical epicyclic frequencies $v_{\mathrm{r}}, v_{\theta}$ (for their explicit definition in the more general Kerr-Newman spacetime see Aliev \& Galtsov (1981)). Stuchlík et al. (2007a) showed that for $a_{*} \rightarrow 1$ the humpy frequency and the epicyclic frequencies evaluated at the "humpy radius" reach asymptotic, i.e. almost spin-independent, commensurable values in the ratios $v_{\theta}: v_{\mathrm{r}}: v_{\mathrm{h}} \simeq 11: 3: 2$. Moreover, in the region with a positive slope of $\mathcal{V}^{(\varphi)}$ the orbits of commensurability between vertical and radial epicyclic frequencies $r_{3: 1}$ and (for $a_{*} \gtrsim 0.996$ ) $r_{4: 1}$ are located (see Aschenbach 2004; Stuchlík et al. 2007a), supporting the idea of a connection between the humpy profile of $\mathcal{V}^{(\varphi)}$ and epicyclic motion of particles. In general, it is also possible to expect oscillations of the disc excited by the orbital velocity hump; the concrete physical mechanism is, however, unknown.

A model in which the resonance between the hump-induced and epicyclic motion is assumed, the so-called Extended Orbital Resonance Model (ExORM), was applied to the X-ray variable Galactic black hole GRS $1915+105$ in which at least five highfrequency QPOs with frequencies $v_{1}=27 \mathrm{~Hz}$ (Belloni et al. 2001), $v_{2}=41 \mathrm{~Hz}, v_{3}=67 \mathrm{~Hz}$ (Morgan et al. 1997; Strohmayer 2001), $v_{4}=113 \mathrm{~Hz}, v_{5}=167 \mathrm{~Hz}$ (Remillard 2004) had been observed. The model predicts a near-extreme spin of the central black hole, $a_{*}=0.9998$, which is in agreement with results given by spectral continuum fits, $a_{*}>0.98$, presented by McClintock et al. (2006), and the black-hole mass $M_{\mathrm{BH}} \sim 14.8 M_{\odot}$, being well inside the interval $(14.0 \pm 4.4) M_{\odot}$ given by other observational methods (Remillard \& McClintock 2006). The results are partly summarized in Fig. 3 and fully described in our previous paper (Stuchlík et al. 2007b).

\section{Mass estimate of the XTE J1650-500 black hole}

As mentioned above, the very broad Fe-line observed in XTE J1650-500 is usually interpreted as a signature of 


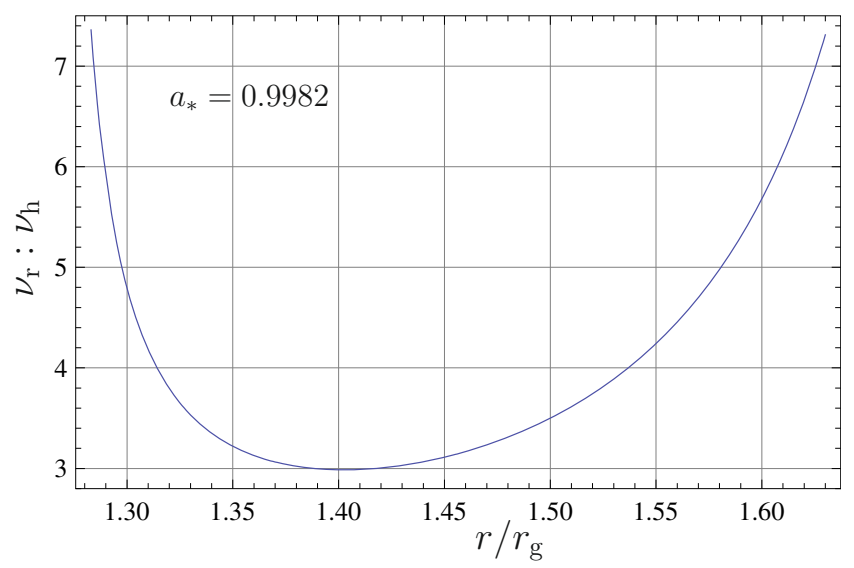

Fig. 2. Ratio of the radial epicyclic frequency and the humpy frequency for equatorial geodesic orbits around the Kerr black hole with spin $a_{*}=$ 0.9982 .

a near-extreme Kerr black hole present in the system ${ }^{3}$. If the source really contains the black hole spinning close to the "Thorne limit" $a_{*} \simeq 0.998$, the effects of a non-monotonic orbital velocity profile may be seen there.

For test-particle (geodesical) discs and the spin $a_{*}=0.9982$, the ratio of the radial epicyclic frequency and the characteristic "humpy frequency", both computed at the same radius, is presented in Fig. 2. Of special interest are the ratios given by small integers like 3:1, 4:1, and 5:1, which can play a role in resonances between hump-induced and epicyclic oscillations. We assume that the most probable mechanism is forced resonance, however, other kinds of resonances are not completely excluded. The most relevant is the 3:1 ratio, which can be excited through the superharmonic resonance due to some non-linearity of the 3 -rd order that can be assumed in the system (Nayfeh \& Mook 1995). Moreover, the strength of the resonance decreases with rising order of the non-linearity (Landau \& Lifshitz 1976), being stronger for the $3: 1$ ratio than for the $4: 1$ or 5:1 ratio. Further, the $3: 1$ resonant radii are located at orbits very close to the "humpy radius" $r_{\mathrm{h}} \simeq 1.417 r_{\mathrm{g}}$, i.e., the orbit where the characteristic "humpy frequency" reaches its maximal value given by Eq. (2), and where the Aschenbach effect is the strongest. Locations of resonant radii 3:1, 4:1, and 5:1 can be inferred from Fig. 2 and are explicitly given in Table 1 .

Now we identify the strong $250 \mathrm{~Hz}$ QPO with the radial epicyclic frequency at a given resonant radius and compute the corresponding black-hole mass. At the preferred "humpy radius", which almost coincides with the outer resonant orbit of the 3:1 resonance, the radial epicyclic frequency reaches $1275\left(M / M_{\odot}\right)^{-1} \mathrm{~Hz}$, which gives the mass of the Kerr black hole $M_{\mathrm{BH}} \simeq 5.1 M_{\odot}$. Estimates corresponding to other resonant radii are given in Table 1 .

\section{Conclusions}

Our suggested application of the ExORM to observed quasiperiodic variability in the X-ray transient XTE J1650-500, like other strong-gravity orbital models of high-frequency QPOs, e.g. the "Relativistic Precession Model" of Stella et al. (1999) or the orbital resonance model of Kluźniak \& Abramowicz (2001), is

\footnotetext{
3 Note that some other alternatives were proposed to explain such broadness, e.g. Done \& Gierliński (2006).
}

Table 1. Location of resonant orbits (inner, outer) corresponding to ratios $v_{\mathrm{r}}: v_{\mathrm{h}}=3: 1,4: 1$, and 5:1 for the Kerr black hole $\left(a_{*}=0.9982\right)$ schematically given in Fig. 2. The next two columns show massestimates for the black hole in XTE J1650-500, if the radial epicyclic frequency at each orbit is identified with the observed $250 \mathrm{~Hz}$ QPO.

\begin{tabular}{ccccc}
\hline \hline$v_{\mathrm{r}}: v_{\mathrm{h}}$ & $r_{\text {in }} / r_{\mathrm{g}}$ & $r_{\text {out }} / r_{\mathrm{g}}$ & $M_{\text {in }} / M_{\odot}$ & $M_{\text {out }} / M_{\odot}$ \\
\hline $3: 1$ & 1.388 & 1.417 & 4.7 & 5.1 \\
$4: 1$ & 1.314 & 1.537 & 3.3 & 6.6 \\
$5: 1$ & 1.298 & 1.581 & 3.0 & 7.0 \\
\hline
\end{tabular}

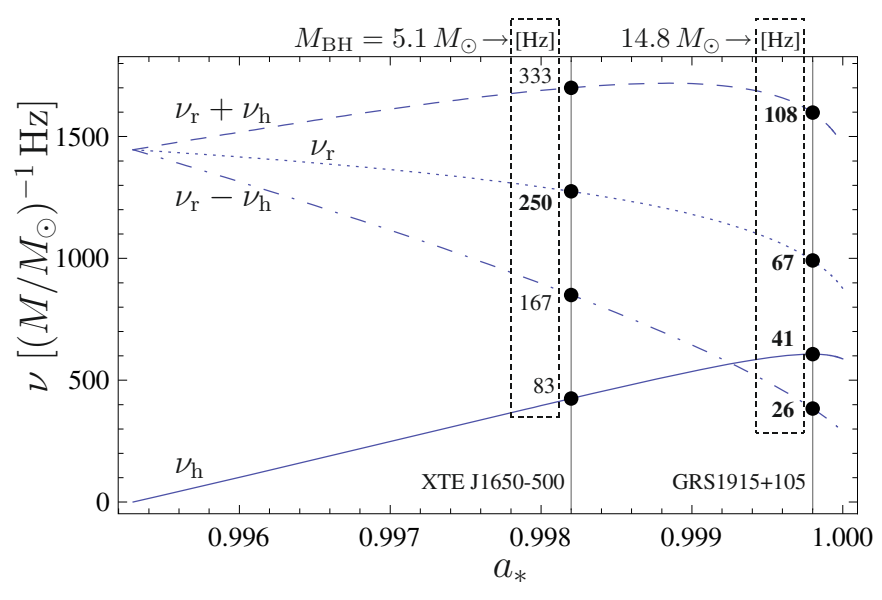

Fig. 3. Spin dependence of the humpy and radial epicyclic frequency, $v_{\mathrm{h}}$ and $v_{\mathrm{r}}$, and their combinational frequencies $v_{\mathrm{r}} \pm v_{\mathrm{h}}$. All frequencies are calculated at the same radius, where the rate of change of the orbital velocity in terms of the proper radial distance reaches the maximal positive value for a given $a_{*}$. The values of frequencies corresponding to the black-hole mass and spin implied by suggested explanations of X-ray variability in the XTE J1650-500 and GRS 1915+105 binary black-hole systems, are given. The bold-faced values can be identified with the observed ones, referred to as QPOs for a given source.

able to give estimates for the mass and spin of the Kerr black hole, if more than one frequency or one of black-hole parameters are known. Of course, the employed ideas are relevant only if the source harbours a near-extreme Kerr black hole, because only in this case does the LNRF-related orbital velocity of the accretion disc reveal non-monotonic behavior in its inner part close to the ISCO.

Following the spectral and timing properties we assume that the spin of the Kerr black hole in XTE J1650-500 is equal to the "Thorne limit" $a_{*}=0.9982$ for the electron-scattering photon emission law, and that the observed high-frequency QPO at $250 \mathrm{~Hz}$ corresponds to the epicyclic motion in the orbital plane, excited by the orbital velocity hump. The first assumption about the spin relies on analyses of the broad $\mathrm{Fe} \mathrm{K} \alpha$ line (Miller et al. 2002; Miniutti et al. 2004) which suggest that the spin is close to the "Thorne limit". The second assumption about the nature of the $250 \mathrm{~Hz}$ QPO is model-dependent. Further we expect the existence of resonances between the hump-induced and epicyclic motions. Under these assumptions we evaluated ratios of the radial epicyclic frequency and the characteristic humpy frequency in the region of a positive radial gradient of $\mathcal{V}^{(\varphi)}$, and found particular resonant radii. The most promising seems the resonance $v_{\mathrm{r}}: v_{\mathrm{h}} \sim 3: 1$, since it takes place at the "humpy radius" where the positive rate of change of the orbital velocity with the proper radial distance is maximal and its strength is much higher in comparison to resonances with a $4: 1$ or $5: 1$ frequency ratio 
(Landau \& Lifshitz 1976). In the case of the 3:1 ratio at the humpy radius the inferred mass of the black hole in XTE J1650500 is $5.1 M_{\odot}$, which is well below the upper limit of $7.3 M_{\odot}$ given by Orosz et al. (2004). The humpy frequency and corresponding combinational frequencies $v_{\mathrm{r}} \pm v_{\mathrm{h}}$ that naturally exist in non-linear systems are given in Fig. 3. Recall that some broad high-frequency features near frequencies of $80 \mathrm{~Hz}$ (Kalemci et al. 2003) and $168 \mathrm{~Hz}$ (Homan et al. 2003) were reported, and could be identified with $v_{\mathrm{h}}$ and $v_{\mathrm{r}}-v_{\mathrm{h}}$, respectively ${ }^{4}$.

The general character of the humpy frequency, characterizing the "Aschenbach effect" in accretion discs, leads us to expect that the real frequency of any process connected with the orbital velocity hump differs from the maximal value for $v_{\mathrm{h}}$, and, in general, is not fixed to any particular value, depending, e.g., on the accretion rate and internal processes in the inner part of the disc. For a $\left(5.1 M_{\odot}, 0.9982\right)$ Kerr black hole the range of radii for which the humpy frequency reaches values between $50 \mathrm{~Hz}$ and $83 \mathrm{~Hz}$ is emphasized in Fig. 1. This frequency interval, containing the observed broad high-frequency features in XTE J1650500 , covers almost the whole region with a positive radial gradient of $\mathcal{V}^{(\varphi)}$.

The main goal of this note is to show that the observed spectral and timing properties of the X-ray transient XTE J1650-500 black hole could be interpreted in terms of the ExORM, giving the possibility to estimate the mass of the black hole. Nevertheless, the presented results should not be considered definite, rather they should be taken as another contribution to the discussion about the character of the black hole in XTE J1650-500, supporting some previously published results and emphasizing the possible existence of the new effect in accretion discs around near-extreme Kerr black holes discovered by Aschenbach four years ago.
Acknowledgements. The authors acknowledge useful discussions with Gabriel Török. The work has been done as a part of the research project MSM 4781305903 financed by the Czech Government.

\section{References}

Aliev, A. N., \& Galtsov, D. V. 1981, Gen. Relativity Gravitation, 13, 899 Aschenbach, B. 2004, A\&A, 425, 1075

Bardeen, J. M., Press, W. H., \& Teukolsky, S. A. 1972, ApJ, 178, 347 Belloni, T., Méndez, M., \& Sánchez-Fernández, C. 2001, A\&A, 372, 551 Done, C., \& Gierliński, M. 2006, MNRAS, 367, 659

Homan, J., Klein-Wolt, M., Rossi, S., et al. 2003, ApJ, 586, 1262

Kalemci, E., Tomsick, J. A., Rothschild, R. E., et al. 2003, ApJ, 586, 419

Kluźniak, W., \& Abramowicz, M. A. 2001, Acta Phys. Polon. B, 32, 3605

Landau, L. D., \& Lifshitz, E. M. 1976, Course of Theoretical Physics Vol. 1: Mechanics (Elsevier Butterworth-Heinemann), 3rd edn

Laor, A. 1991, ApJ, 376, 90

McClintock, J. E., Shafee, R., Narayan, R., et al. 2006, ApJ, 652, 518

Miller, J. M., Fabian, A. C., Wijnands, R., et al. 2002, ApJ, 570, L69

Miniutti, G., Fabian, A. C., \& Miller, J. M. 2004, MNRAS, 351, 466

Morgan, E. H., Remillard, R. A., \& Greiner, J. 1997, ApJ, 482, 993

Nayfeh, A. H., \& Mook, D. T. 1995, Nonlinear Oscillations (John Wiley \& Sons, Inc.)

Orosz, J. A., McClintock, J. E., Remillard, R. A., \& Corbel, S. 2004, ApJ, 616, 376

Remillard, R. A. 2004, AIPC, 714, 13

Remillard, R. A. \& McClintock, J. E. 2006, ARA\&A, 44, 49

Stella, L., Vietri, M., \& Morsink, S. M. 1999, ApJ, 524, L63

Strohmayer, T. E. 2001, ApJ, 554, L169

Stuchlík, Z., Slaný, P., \& Török, G. 2004, in Proceedings of RAGtime 4/5: Workshops on black holes and neutron stars, Opava, 14-16/13-15 Oct. 2002/03, ed. S. Hledík \& Z. Stuchlík (Opava: Silesian University in Opava)

Stuchlík, Z., Slaný, P., \& Török, G. 2007a, A\&A, 463, 807

Stuchlík, Z., Slaný, P., \& Török, G. 2007b, A\&A, 470, 401

Stuchlík, Z., Slaný, P., Török, G., \& Abramowicz, M. A. 2005, Phys. Rev. D, 71, 024037

Thorne, K. S. 1974, ApJ, 191, 507

\footnotetext{
4 At the 10th HEAD meeting held in Los Angeles, California from March 31st to April 3rd, 2008, Shaposhnikov \& Titarchuk presented another estimate of the mass of the XTE J1650-500 black hole obtained by Quasi Periodic Oscillation - Spectral Index scaling method of (3.8 \pm $0.5) M_{\odot}$, which is also in good agreement with dynamical measurements and remarkably close to the expected neutron star maximum mass.
} 